ARTICLE

\title{
Biomimetic design of iridescent insect cuticles with tailored, self-organized cholesteric patterns
}

Adriana Scarangella ${ }^{1}$, Vanessa Soldan ${ }^{2} \&$ Michel Mitov (iD ${ }^{1 凶}$

Replicating biological patterns is promising for designing materials with multifaceted properties. Twisted cholesteric liquid crystal patterns are found in the iridescent tessellated cuticles of many insects and a few fruits. Their accurate replication is extremely difficult since discontinuous patterns and colors must coexist in a single layer without discontinuity of the structures. Here, a solution is demonstrated by addressing striped insect cuticles with a complex twisted organization. Geometric constraints are met by controlling the thermal diffusion in a cholesteric oligomer bilayer subjected to local changes in the molecular anchoring conditions. A multicriterion comparison reveals a very high level of biomimicry. Proof-of-concept prototypes of anti-counterfeiting tags are presented. The present design involves an economy of resources and a high versatility of chiral patterns unreached by the current manufacturing techniques such as metallic layer vacuum deposition, template embossing and various forms of lithography which are limited and often prohibitively expensive.

\footnotetext{
${ }^{1}$ Centre d'Elaboration de Matériaux et d'Etudes Structurales, CEMES, CNRS, Toulouse, France. ${ }^{2}$ Centre de Biologie Intégrative, CBI, Microscopie Electronique Intégrative, METi, CNRS, University of Toulouse, Toulouse, France. ${ }_{\text {email: mitov@cemes.fr }}$
} 
A

huge diversity of patterns and colors is found in nature and at different length scales ${ }^{1,2}$, which inspire the design of materials for addressing modern challenges in bioengineering, energy capture and conversion, sensing, communication or data processing ${ }^{3}$. However, current biomimetic materials rarely capture the range of structural complexity observed in natural materials, as acknowledged in recent reviews ${ }^{3-5}$. In addition, a majority of the so-called biomimetic materials rely on manufacturing processes that have scalability limitations.

The biomimetic design of performance materials mimicking the structure of insect cuticles is still in its infancy because the design tools necessary for the control of nano- and microscale patterns with a high level of versatility are still pending6. In the present work, we approached the problem by focusing on tessellated (patterned) and iridescent cuticles with a complex twisted organization of chitin fibres. The cuticles of insects have been pivotal to their successful adaptation to the external environment. Both the exocuticle and endocuticle involve twisted cholesteric liquid crystal (CLC) organization of pseudolayers of chitin macromolecules ${ }^{7}$ (Fig. 1a), which causes a spatially varying index of refraction. The term pseudolayers is used instead of layers because the CLC structure is not a layered system. A stack of layers with an orientational order of rod-like molecules in each plane is often drawn to represent the CLC structure. However, these layers have no physical basis and this representation is only a guide for the observer, as the rod-like molecules have no positional order. Each layer behaves like a uniaxial anisotropic medium with the slow axis parallel to the molecules and the fast axis perpendicular to them when the CLC structure is modelled as a layered system. These two axes twist regularly by a constant angle and without discontinuity from one layer to the next. When light propagates through a CLC in the Bragg regime, the medium behaves like a multilayer system, producing interferential colors. The orientation of the molecular director (the orientation of the elongated chitin fibres depicted in Fig. 1a) and thus the optical axis are modulated helicoidally, rotating in a plane that is perpendicular to the helical axis.

For circularly polarized light propagating along the helix axis with the same handedness as the helix, a CLC exhibits a Bragg reflection band over the wavelength range of $\left(n_{\mathrm{o}}-n_{\mathrm{e}}\right) p$, where $n_{\mathrm{o}}$ a
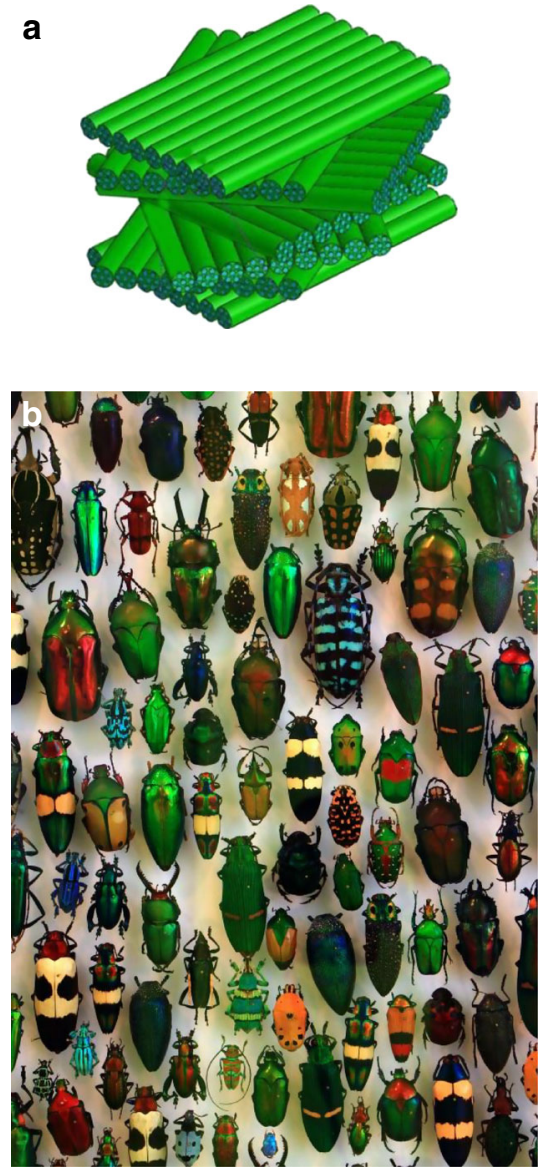

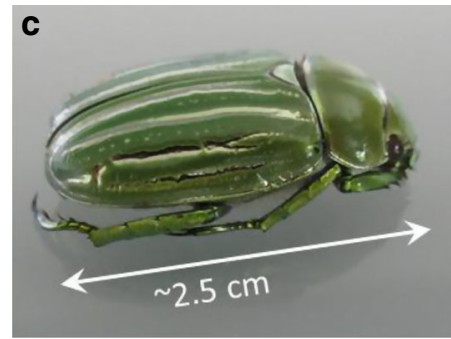

d

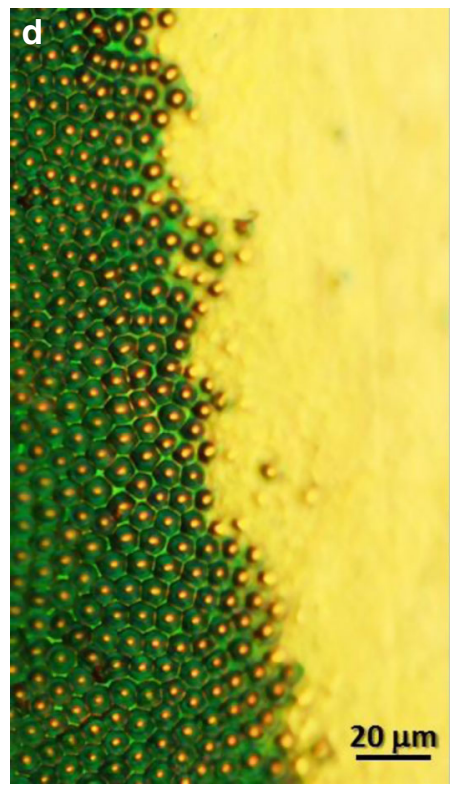

e

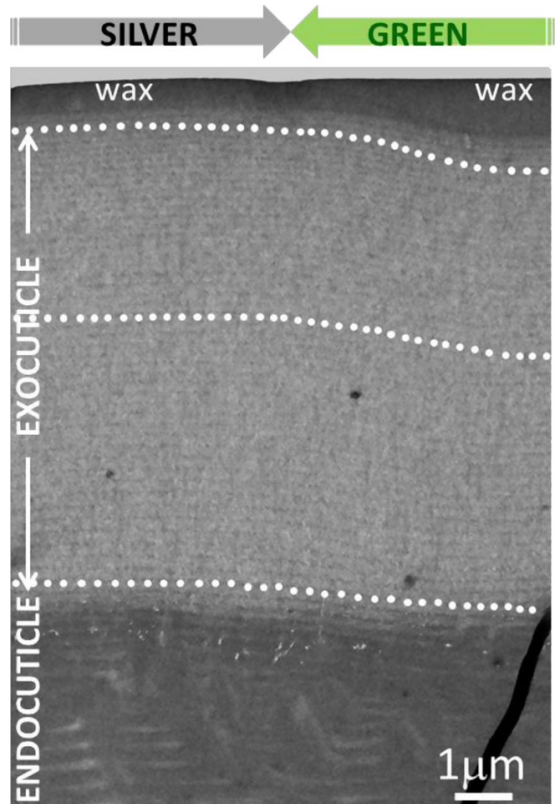

f

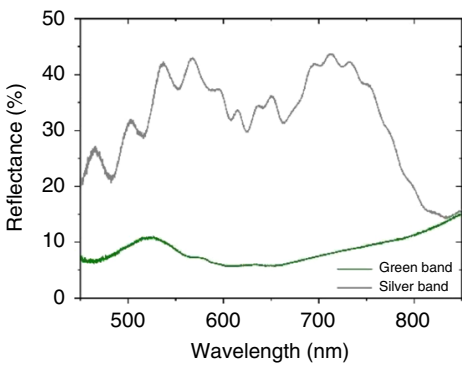

Fig. 1 Lessons from nature. a In the insect cuticle, chitin macromolecules form fibrils that wrap with proteins and assemble into fibres, which assemble into bundles. The bundles arrange themselves parallel to each other and form pseudolayers. Finally, the chitin fibre-protein layers stack into a twisted plywood Bouligand structure ${ }^{7}$. The structure is not a discrete multilayer system; instead, a continuous twist occurs along the axis perpendicular to the fibres, which interpenetrate from one pseudolayer to the next. For this reason, the term pseudolayer is preferred over layer. A $360^{\circ}$ rotation of the fibre orientation defines the helical pitch. (Adapted from ref. ${ }^{75}$. Creative Commons license.) b The insect world includes a very large variety of tessellated (patterned) cuticles with bumps, pits, pixels, bands, spots or patches and a diversity of structural colors (Staatliches Museum für Naturkunde, Karlsruhe, Germany. Image by H. Zell. Creative Commons license). c Two-band iridescent cuticle of Chrysina gloriosa. $\mathbf{d}$ Green and silver bands at their interface as observed by optical microscopy (reflection mode, unpolarized light). e TEM images of transverse structures at the interface between green and silver bands and close to the top surface. White dots underline the stripes in the fingerprint texture. The transition between curved and straight stripes is observed to be very gentle. The last part of the cuticle consists of a wax layer ${ }^{76}$, which may serve to restrict water loss and prevent desiccation ${ }^{77}$. $\mathbf{f}$ Reflection spectra of green and silver bands. 
and $n_{\mathrm{e}}$ are the ordinary and extraordinary refractive indices and $p$ is the helical pitch ${ }^{8}$. The central wavelength $\lambda_{0}$ is: $\lambda_{0}=n p$, where $n=\left(n_{\mathrm{o}}+n_{\mathrm{e}}\right) / 2$ is the average refractive index.

In biomimicry, many of the same species come up': geckos, spiders, and butterflies. The latter are dominant in the field of photonic structures. Air layers and pillar-supported chitinous layers alternate in the twist-free, multilayer structure of butterfly wings, which leads to optical interference ${ }^{10}$. There are many replication methods, which are based on three concepts that use wings as templates ${ }^{11}$ : coating, modification and filling. The morphology of the replicas can be hard to control. The choice of the method depends on the requirements of the situation.

Biomimetic materials aiming at reproducing the cuticles of beetles as closely as possible are especially rare. They have addressed the untextured, gold scarab beetle Plusiotis resplendens $^{12,13}$. The samples consist in the superposition of three layers: a nematic LC layer, which acts as a half-wave retardation plate, is inserted between two left-handed cholesteric layers. The fabrication methods used a UV-crosslinked elastomer ${ }^{12}$ or chiral cellulose nanocrystals after water evaporation ${ }^{13}$. In the latter case, a non-uniformity of the reflection colors at the surface of the sample resulting from the evaporation process is reported. These three-layer systems present interfaces. When optical performance is desired, interfaces likely add parasitic reflections and light scattering to Bragg reflections. Besides, the cuticle of P. resplendens is interface-free: the cholesteric-nematic interfaces do not indeed exhibit any discontinuity ${ }^{14}$.

In contrast to nontextured reflective cuticles, tessellations as found in the cuticles of many insects ${ }^{15-17}$ (Fig. 1b), and a few fruits ${ }^{18,19}$ are challenging to duplicate via a biomimetic design. First, the pitch length and the helical axis orientation must be position-dependent across the material, and formation of periodic patterns at its surface is simultaneously required. The primary challenge consists of finding the design rules to manufacture a single-piece material that presents variable physical properties without resorting to an accumulation of layers and a juxtaposition of patterns. Second, tessellated cuticles are associated with the concept of multiscale structures. Biomimetic materials must thus include anisotropic architectures at the nanoscale, micrometre scale and macroscale. Third, the double versatility of colors and patterns must ideally be achieved through a simple and single stage to make the self-organization process closer to morphogenesis. Finally, an optimal use of resources has to be targeted, as in nature: reducing the number of materials to be implemented since living organisms use only a few chemical elements $(\mathrm{C}, \mathrm{H}, \mathrm{O}$, $\mathrm{N}, \mathrm{P}$ and $\mathrm{S}$, typically) to self-organize into a multitude of complex structures; avoiding metallic nanoparticles and metallic layers to realize an environmentally responsible approach; and considering the recyclability of materials from the perspective of mass-market production.

In this paper, we approach the problem of the high-fidelity capture of the structural complexity observed in nature by focusing on patterned, iridescent carapaces of insects with a complex twisted organization of chitin fibres. At a small scale (the micrometre or submicrometre scale), the helical pitch and orientation of the helical structure depend on the position in three dimensions, and the formation of macroscopic periodic patterns is required. We used LC oligomers to mimic biological LCs. We attempted to reproduce the textural, structural and color properties of these biological LCs at several length scales. We made optimal use of resources and stages during the fabrication procedure, in the spirit of eco-design. By means of a single sequence based on self-organization, precise control of a singlepiece sample structure composed of two different colored patterns with the same unique pitch gradient was enabled. The functionalities of both materials are relevant to optical communication and camouflage. In addition to this set of conceptual advances, we present a concrete use of the material for advanced optical tags in cryptography.

\section{Results}

Lessons from nature. With the previous objectives in mind, we focus on the emblematic case of the scarab beetle Chrysina gloriosa (Fig. 1c), which is found from southwestern North America to Central America in pine, pine-oak, and juniper forests. The cuticle of C. gloriosa exhibits green and silver bands with selective and broad light reflections, respectively. The green bands exhibit a cellular polygonal texture in optical microscopy images, whereas the silver bands exhibit a non-patterned planar texture (Fig. 1d). Previous studies on the cuticle of C. gloriosa have reported the following: the cellular structure of the concentric rings in the green bands, as established through TEM investigations ${ }^{20}$ or fluorescence confocal microscopy ${ }^{21}$; the variation in the optical properties with the incidence angle, as established through Mueller matrix spectroscopic ellipsometry ${ }^{22,23}$; the existence of an array of multiwavelength micromirrors in the green bands ${ }^{24}$; and the generation of self-healing Bessel beams from polygonal cells ${ }^{25}$.

In the following, we set out the lessons learned from nature about the structure and optical response of both bands.

Cross-sectional transmission electron microscopy (TEM) views of the green and silver bands show the typical cholesteric fingerprint (stripe) texture (Fig. 1e). The axis of the helix lies in the plane of the image. The fingerprint texture of the (unlayered) cholesteric structure appears as a network of alternating bright and dark stripes. This periodic network thus arises from periodic modulation, along the helix axis, of the orientation of the molecular director (the orientation of the elongated chitin fibres depicted in Fig. 1a) and the refractive index. The helical axis is perpendicular to the stripes. The distance between two identical stripes is related to the half pitch of the twisted organization of the chitin fibres. For the green bands, the cuticle displays curved stripes in the upper part of the exocuticle, which means that the orientation of the helical axis is spatially changing, and parallel stripes in most of the endocuticle region. For the silver bands, the stripes are parallel in the whole chitinous part of the cuticle, the orientation of the helical axis is fixed. For both bands, a pitch gradient occurs from the top of the exocuticle to the bottom of the endocuticle (Supplementary Fig. 1).

Before proceeding to the analysis of the optical spectra, a preliminary comment must be made here regarding the fact that between 0 and $50 \%$ of the unpolarized incident light is reflected or transmitted, which is true for all of the reflection and transmission spectra presented in this study. Unlike common mirrors, which reverse the polarization of the light they reflect, the cholesteric planar texture reflects circularly polarized light with the same handedness as that of the twist of the helix, while light with the other polarization is transmitted ${ }^{26}$. This behavior is referred to as the polarization-selectivity rule, which is valid only at normal incidence. Consequently, up to $50 \%$ of the ambient unpolarized light at the selected wavelength can be reflected. The other component $(50 \%$ of the ambient unpolarized light at the selected wavelength) is transmitted. Only first-order Bragg reflection is possible at normal incidence. All orders of reflection may occur at oblique incidence or when the helical structure is tilted, and the reflected or transmitted light is elliptically polarized.

The reflectance spectrum ascribed to the silver band in Fig. If exhibits a broadband reflection from 450 to $800 \mathrm{~nm}$ with a variable reflectance. This broad band can be identified from the pitch gradient shown in the TEM micrograph of a cross section (Supplementary Fig. 1b) and the related measurements, which 
give a pitch $\mathrm{p}$ varying from $\sim 280$ to $900 \mathrm{~nm}$ (Supplementary Fig. 2a). By taking $n=1.63$ for the value of the average refractive index of the chitinous part of the cuticle of C. gloriosa (in ref. 14 (p. 214), the ordinary and extraordinary indices are set to 1.59 and 1.68 for Plusiotis gloriosa (a synonym now invalid for $C$. gloriosa), which leads to a mean refraction index equal to $\sim 1.63$.) and using the Bragg law at normal incidence, $\lambda_{0}=n p$, as given in the introduction, we obtain a wavelength band of $\sim 455-1470 \mathrm{~nm}$ (the measurements in Fig. If stop at $850 \mathrm{~nm}$ because of the technical limitations of the spectrophotometer). The silver band thus plays the role of a flat metallic reflector operating over the visible spectrum and into the infrared spectrum. The oscillations, or ripples, modulating the reflection spectrum in Fig. If can be attributed to longitudinal interferences associated with the existence of a singular region named the emergence line between the exo- and endo-cuticles (i.e., a trough in the periodicity variation), as observed by SEM and discussed in ref. ${ }^{24}$. The presence of a ripple structure superimposed on a broadband plateau is also present in the spectrum of the gold cuticle of the scarab beetle Chrysina aurigans ${ }^{27,28}$.

The reflectance spectrum ascribed to the green band exhibits a broadband reflection from 450 to $800 \mathrm{~nm}$. The pitch gradients in the silver and green bands occur in ranges of comparable amplitudes, as shown in Supplementary Fig. 2a. However, for the green band, the reflectance is very low (between 8 and 10\%) from 450 to $800 \mathrm{~nm}$, with a characteristic green peak at $520 \mathrm{~nm}$, because the green bands act as green diffusers. Diffuse reflection occurs when light is reflected at many different angles rather than at just one angle as in the case of specular reflection. The green bands are green diffusers because the arrangement on a curved surface of polygonal cells, with curved and concave stripes (Fig. 1e and Supplementary Fig. 1a), with a spatially varying helical axis, significantly broadens the angular distribution of reflection. Both kinds of curvature (the surface of the cuticle, and the orientation of the helical axis inside each cell) remove the angular dependence of the reflection wavelength normally associated with Bragg reflection when the cholesteric material is a flat film with a pattern-free planar texture. The result is a nearly angle-independent greenish appearance of the beetle at long viewing distances, which may enable the avoidance of detection by predators in green foliage. The disruptive green-silver patterning is a good match for the habitat of C. gloriosa since the foliage of the juniper tree is green with white resin flecks. This situation might provide the insect with efficient camouflage ${ }^{29}$.

It should be noted that the reflective and absorptive properties of the wax layer at short wavelengths may influence the shape of the reflection spectrum at the beginning of the visible region, as discussed in the case of Chrysina aurigans ${ }^{30}$.

To summarize, Fig. 2a shows a $3 \mathrm{D}$ view that displays the color and structure properties to be simultaneously addressed in the goal of biomimetic design. The biomimetic material must be a monolayer; exhibit a disruptive character of green and silver colors; display the continuity of the twisted structures in all directions (no disruption in the pitch distribution and in the orientation of the helical axis from region to region) with, in the upper part of the film, an alternate mix of variable and fixed orientations of the helical axis; include a pitch gradient related to reflection colors ranging from green to near IR; and exhibit pitch variations in similar ranges.

Biomimetic design. Recent successes in utilizing soft materials for the design of advanced photonic structures promise compelling opportunities, but they rarely capture the range of structural complexity observed in natural optical materials ${ }^{31}$. Using synthetic LC materials for biomimetic purposes is an underutilized solution in the toolbox of researchers and engineers, partly due to challenges in fabrication, integration, and structural control at the nanoscale and microscale of soft components. However, LCs are first-rate candidates because they offer anisotropic self-organization, multiscale architectures, and a diversity of materials, such as fluids, polymers or gels, that can be inserted between two solid or flexible substrates or handled as free-standing films.

The design procedure we propose uses vitrifiable cholesteric oligomers with a siloxane backbone. The rapid cooling of samples below the glass-transition temperature, from the viscous cholesteric phase to room temperature, prevents crystallization and preserves the cholesteric structure and colors. Polysiloxanes form a group of materials with unusual properties, which may include high thermal stability, low surface energy and a low glasstransition temperature ${ }^{32}$. The present biomimetic design takes advantage of these properties. LC polysiloxane oligomers from Wacker Chemie $\mathrm{GmbH}$ were used ${ }^{33}$ (Supplementary Fig. 3). The molecule is a siloxane cyclic chain with two types of side chains attached via spacers: an achiral mesogen and a (chiral) cholesterol-bearing mesogen ${ }^{34}$. When used to coat a glass or plastic substrate, this compound exhibits typical iridescent colors that range from blue to red with the tuning of the molar percentage of chiral mesogens in the oligomer molecule, which can vary from 31 to $50 \%$. LC polysiloxane oligomers have been the subject of various studies, including basic investigations of their reflective properties ${ }^{35}$, the fabrication of optical notch filters and variable bandwidth reflectors ${ }^{36,37}$, low-frequency mechanical relaxation measurements of molecular motions near the glasstransition temperature ${ }^{38}$, the color gamut of planar films and flakes based on colorimetry ${ }^{39}$, the electro-optical behavior of cholesteric flakes ${ }^{40}$, mechano-optical effects ${ }^{41}$, lasing from dyedoped materials ${ }^{42}$, the self-organization of metallic nanoparticles into various cholesteric textures ${ }^{43,44}$ and the control of the stretching and compression of ultrashort laser pulses ${ }^{45}$.

Polygonal textures, consisting of arrays of micrometre-scale polygonal cells (Supplementary Fig. 4a), can be observed in flat CLC films when the helical axes are strongly tilted with respect to the substrates. This situation can be observed in an open film via hybrid anchoring of molecules, which preferentially align tangentially to the solid substrate on which the CLC is coated (planar anchoring conditions) and perpendicularly to the air interface (perpendicular anchoring conditions). Supplementary Fig. $4 \mathrm{~b}$ shows a three-dimensional representation of the polygonal texture obtained by combining various microscopy methods and provides more information on the formation of the polygonal texture in the materials under study. Polygonal fingerprint textures were first imaged via optical microscopy in large-pitch low-molar-mass CLCs in the early $1970 \mathrm{~s}^{46,47}$. In the mid-1990s, polygonal textures in the film plane and in cross sections were observed via $\mathrm{TEM}^{48}$ and $\mathrm{AFM}^{49,50}$. In the 2010s, investigations of polygonal textures focused on glass-forming oligomers ${ }^{51-57}$ such as those used in the present study, which does not focus on the polygonal texture itself.

The biomimetic design is based on the control of the thermally driven diffusion between one green and one infrared lightreflecting layer accompanied by local changes in the molecular anchoring conditions in specific regions of the film. It involves five stages (Fig. 2b). Stage 1: A cell made of cholesteric films coated on glass substrates facing each other, reflecting the light in the green and IR spectrum, respectively, is assembled at RT. Stage 2: The sandwich cell is heated at $120^{\circ} \mathrm{C}$ for $30 \mathrm{~s}$ to adhere the films together. Stage 3: After quenching of the sample at RT, the upper substrate is removed. Stage 4: Spaced bands of an isotropic polymer, polyvinyl alcohol (PVA), in solution in water are deposited on the free surface of the bilayer film. Stage 5: After drying of the aqueous solution, the bilayer film is heated in the 


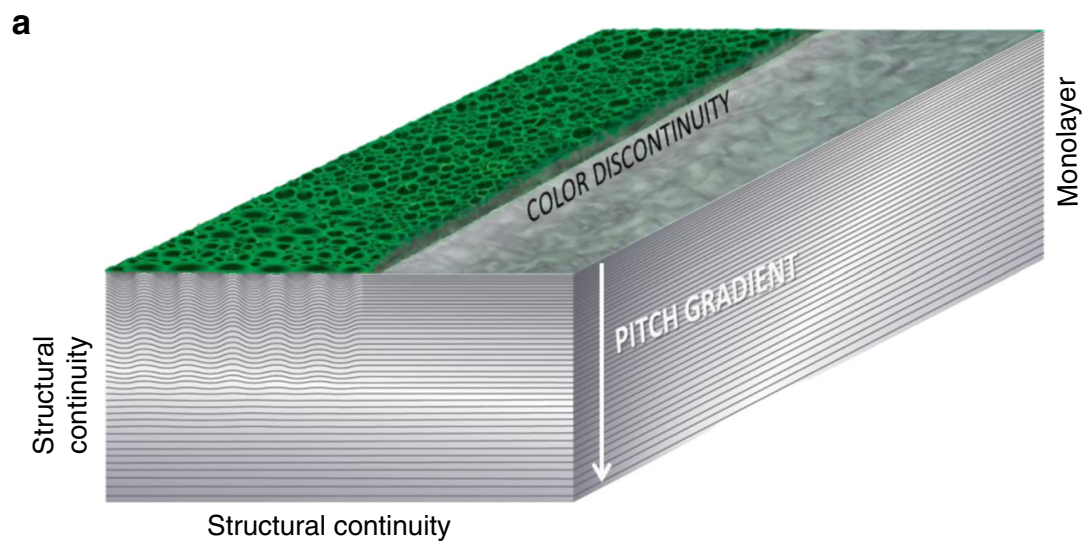

b
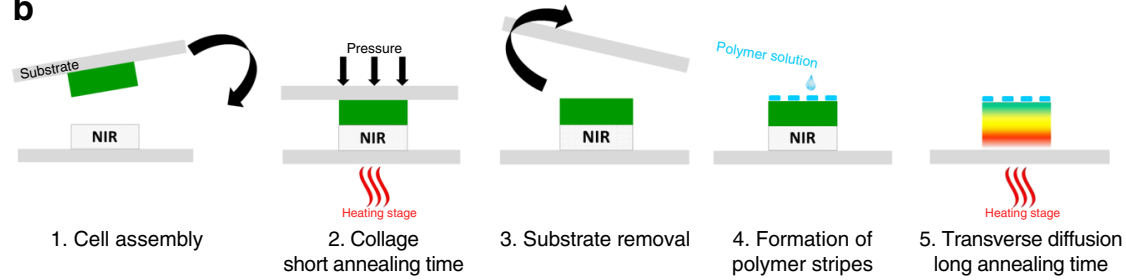

Fig. 2 Fundamental principles to mimic the tessellated cuticle. a Tridimensional two-band structure to be considered. $\mathbf{b}$ Physical design in five stages.

cholesteric phase at $120^{\circ} \mathrm{C}$ for $1 \mathrm{~h}$. Three major events simultaneously occur due to the thermally driven diffusion between the two films in the direction perpendicular to their surfaces: a physical transverse pitch gradient is built as the result of the chemical gradient in the chiral species; the polygonal texture nucleates in the regions in contact with air between the polymer bands (the anchoring of molecules becomes perpendicular in the upper part of these regions), whereas the planar texture is preserved in the regions below the polymer bands (the anchoring of molecules is kept as planar in these regions); and the bilayer system becomes a single layer. Finally, the film is quenched at RT. The structural and optical properties are maintained in the solid glassy film.

The fifth stage of the design is the crucial, one-step, stage. By offering a single sequence based on self-organization, precise control of the sample structure composed of two different colored bands with the same unique pitch gradient is enabled. An association of the alternate green and silver bands was obtained by drawing spaced bands on the free surface of the film before the thermal diffusion began using a pencil dipped in PVA solution. Alternatively, band-shaped adhesive tape could be fixed on the surface of the film, which is then spin-coated with the solution (see Methods). The tape was removed before the solution was completely dried. Many patterns other than bands can thus be drawn, which offers a wide, choice on demand for designing reflective patterns. PVA is commonly used in the field of thermotropic LCs to induce a molecular orientation parallel to the support ${ }^{58}$. Here, it was used to protect specific regions targeted to become silver bands from air. By analogy, it protects the film from texture evolution (planar to polygonal texture transition), while the wax layer visible in Fig. 1e and Supplementary Fig. 1 protects the chitinous part of the cuticle from the external environment, with the difference being that the polymer layer only covers the silver bands, while the wax layer covers the green and silver bands. Finally, the PVA bands can be dissolved in water if required. Patterning techniques, such as metallic layer sputtering deposition ${ }^{31}$, moulding 59 , alignment methods ${ }^{60}$ or lithography ${ }^{61,62}$, would require many stages to achieve the present patterning objectives and would lead to discontinuous layers and structures. In addition, the range of producible patterns, the properties of surface coatings related to these techniques and their durability are supposedly restricted ${ }^{6}$.

Biomimetic samples: texture, structure and optical behavior. Figure $3 \mathrm{a}$ shows the biomimetic samples when coated on a glass substrate or as a free-standing film. The silver bands appear as black because the film (coated on a glass substrate) is placed on a black substrate and transmits light. It is semitransparent: indeed, a cholesteric film reflects at most $50 \%$ of unpolarized ambient light (as explained previously). The perception of colors in the macroscopic images is highly dependent on the ambient lighting conditions and on the viewing angle. This is the reason why, in Fig. 1c, some silver bands of the beetle partially appear as black: the cuticle is curved, and the light reflected by the specular mirrors illuminated by the flash on part of the curved surface is not collected by the camera. On the other hand, all of the green bands are seen as green because the light reflected by the green bands is diffusely reflected. This is a consequence of the existence of an array of polygonal cells in which the orientation of the helical axis is spatially variable. Magnified views of a set of green and silver bands for which the whitish aspect of the silver band is more visible are given in Supplementary Fig. 5. Additional comments regarding the differences in the perceived colors, from the biological to the biomimetic material, will be given below in the discussion of the optical spectra.

Biomimetic samples consist of a single piece that does not include any foreign material. By analogy, solidification by thermal quenching of the viscous material, at the end of the ultimate fifth stage, plays the role (transition from a soft to an hard material) of cuticle sclerotization ${ }^{63}$ after the cuticle has expanded to its final size and shape.

The optical micrograph of the cuticle (Fig. 3b) shows a welldefined boundary between bands, which is more pronounced than in the biological material (Fig. 1d). At the sub-micrometre scale, TEM investigations of cross sections (Fig. 3c) show that the transition between bands is very gentle in the case of the biomimetic material. This set of observations validates the continuity of the transverse structures and the role of the 
a

b
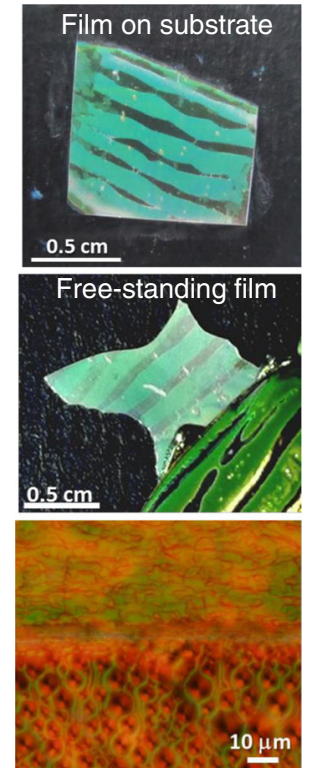

C
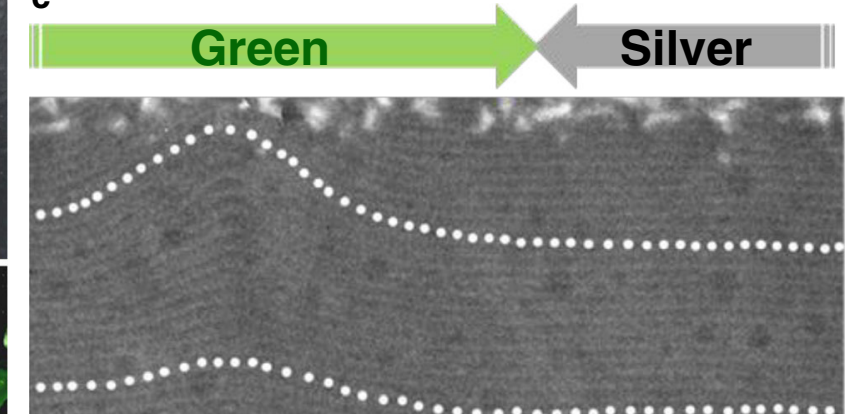

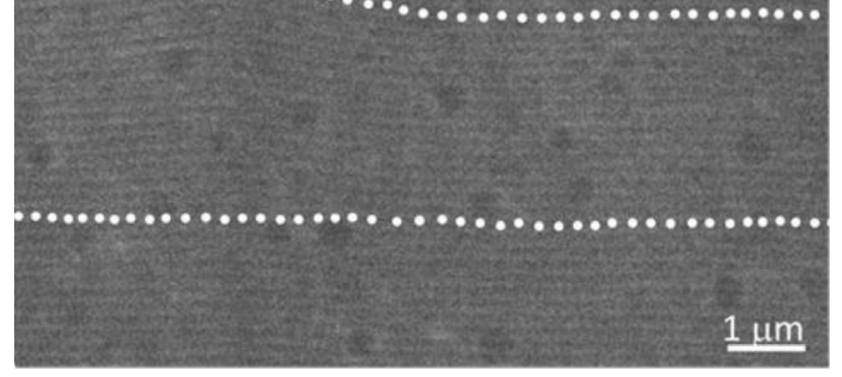

$\mathbf{f}$
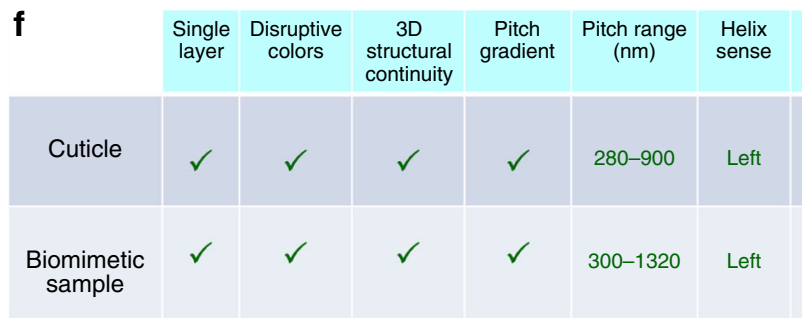

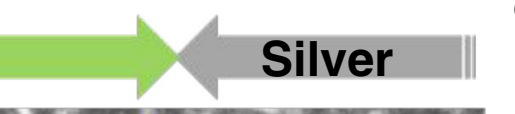

d
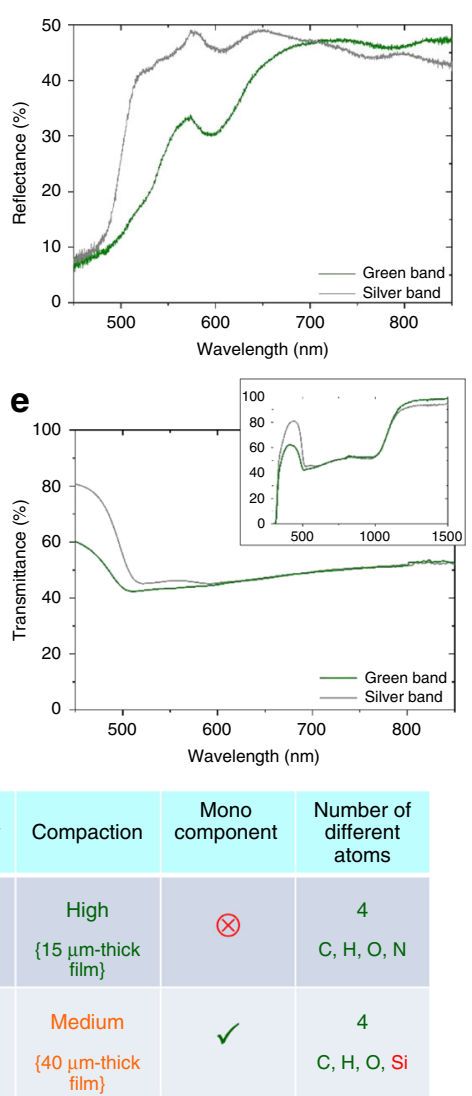

Fig. 3 Features of the biomimetic samples. a Biomimetic samples on a glass substrate and as a free-standing film. b Optical micrograph of the interfacial region between silver (top) and green (bottom) bands (reflection mode, unpolarized light). c TEM image of transverse structures at the interface between green and silver bands and close to the top surface. White dots underline the stripes of the fingerprint texture. The transition between curved and straight stripes is observed to be very gentle. $\mathbf{d}$ Reflectance spectrum of green and silver bands. e Transmittance spectrum of green and silver bands with the same wavelength scale as in $\mathbf{d}$. Inset: the same spectrum displayed with the full operating wavelength band. $\mathbf{f}$ Multicriterion comparative table of features of the cuticle and biomimetic sample. The mentions in green (resp. red) report a similarity (resp. discrepancy). The mention in orange corresponds to an inbetween situation.

orientation of the helical axis-from tilted to perpendicular-in the macroscopic color differences. The texture periodicityrelated to the helical half-pitch-as a function of the film depth is determined from cross-sectional TEM images (Supplementary Fig. 6). The pitch ranges in the biological-ca. $280-900 \mathrm{~nm}$ (Supplementary Fig. 2a)-and biomimetic-ca. 300-1320 nm (Supplementary Fig. 2b)-materials are comparable in their orders of magnitude.

The reflection spectra ascribed to the biomimetic silver and green bands (Fig. 3d) have comparable shapes. Both bands behave as broadband reflectors from $\sim 500$ to $850 \mathrm{~nm}$. This broad wavelength range is comparable to that of the cuticular silver band in Fig. 1f. Some differences in reflectance between the biomimetic silver and green bands are apparent: the spectrum ascribed to the silver band exhibits enhanced reflectance from 500 to $700 \mathrm{~nm}$ because here, the green band continues to exhibit diffuse reflection, whereas the silver band behaves as a specular mirror. However, the reflectance of the biomimetic green band is much more important than that of the cuticular green band. A green peak at $570 \mathrm{~nm}$ is observed for the biomimetic green band, whereas the corresponding peak is located at $520 \mathrm{~nm}$ for the cuticular band. This distinction contributes to the difference in the perceived green reflection color between the biological material and the biomimetic one. Other reasons, of a conceptual nature, may also be invoked to explain why the reflection colors and optical textures of the biomimetic samples are much less vibrant than those of C. gloriosa. First, the chemical compositions of the biomimetic and biological materials are very different. In some beetles, the presence of uric acid in the chitin matrix plays a major role in imparting color by enhancing the birefringence and reflectivity 7,14 . Second, the thin and transparent wax layer may play a role in the perception of colors. It may scatter light and contribute to the shiny appearance of some beetles ${ }^{29}$. In the case of the cuticle of Chrysina boucardi, the overall chromatic effect has been described as deeper in color because the cuticle is covered with wax $^{15}$, a situation that was noted to be evocative of the metal flake paint used on automobiles.

While the opacity of the cuticle prevents the measurement of light transmission, the transmission spectra of the biomimetic sample show a broad bandgap from 500 to $1000 \mathrm{~nm}$ (inset in Fig. 3e). The fact that the plateau begins at $500 \mathrm{~nm}$ is consistent with the sudden increase in reflectance beginning at $\sim 500 \mathrm{~nm}$ in Fig. 3d. The transmittance spectra for both bands are very similar, providing further confirmation that the difference in reflection colors is due to the dual presence of variable and fixed orientations of the helical axis and not to significant pitch variations in the bulk of the material. This broad band is objectified by the pitch gradient shown in the TEM micrograph of a cross section and the related measurements, which give a pitch varying from 300 to $1320 \mathrm{~nm}$ (Supplementary Fig. 2b). By taking $n=1.5$ for the value of the average refractive index of the polysiloxane film ${ }^{64}$ and by using the Bragg law $\lambda_{0}=n p$, at normal 
a

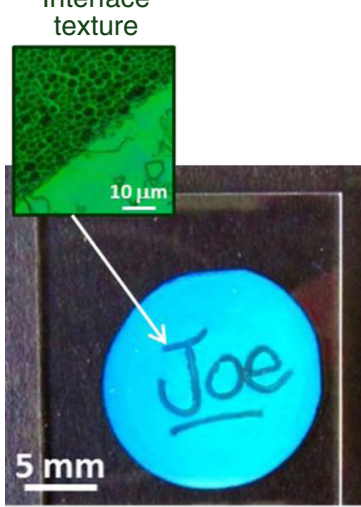

b

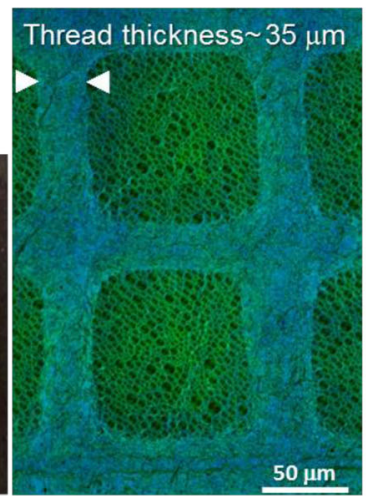

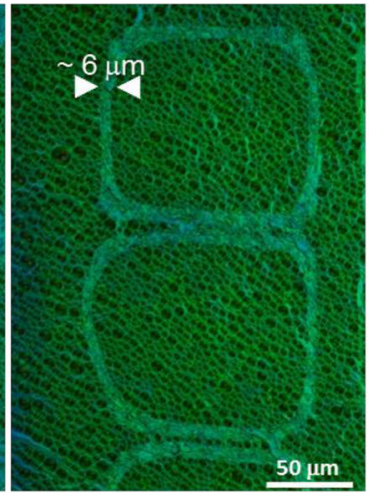

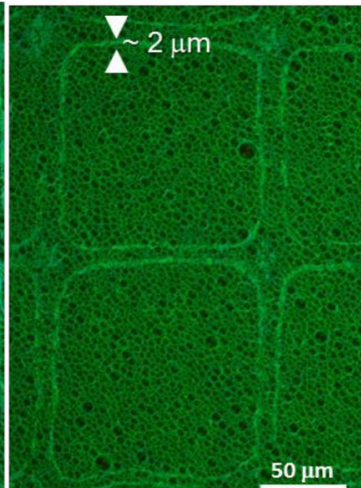

Fig. 4 Practical uses in visual cryptography. Multiscale optical tags with taggants from the a millimetre to $\mathbf{b}$ micrometre scale.

incidence, we obtain a wavelength band of $\sim 450-1980 \mathrm{~nm}$. The wavelengths at the left edge of the Bragg band are comparable: $500 \mathrm{~nm}$, as displayed in Fig. $3 \mathrm{e}$, vs. $450 \mathrm{~nm}$, as calculated. A wavelength discrepancy appears at the right edge: $1000 \mathrm{~nm}$ vs. $1980 \mathrm{~nm}$. We assume that the number of helix turns ascribed to the twist from $666 \mathrm{~nm}(1000 \mathrm{~nm}: 1.5)$ to $1320 \mathrm{~nm}(1980 \mathrm{~nm}: 1.5)$ is too low to allow a contribution to the Bragg band from this part of the pitch gradient. The reflectance indeed depends on the number of pitches (i.e., the number of helix turns), which means that the twist needs to develop within a minimal film thickness to make possible the appearance of a Bragg peak that is detectable under standard spectrophotometry conditions ${ }^{65}$. Our hypothesis is supported by the sudden jump in the half-pitch in the 333-660 nm range for a small sample thickness of $\sim 6 \mu \mathrm{m}$ (vs. the total sample thickness of $40 \mu \mathrm{m}$ ), as shown in Supplementary Fig. 2b. This thickness cannot permit a sufficiently large number of helix turns and the detection of related Bragg reflections.

Multicriterion comparison. A criterion-by-criterion comparative table of the cuticle and biomimetic sample is given in Fig. $3 \mathrm{f}$. Eight of twelve criteria are rated as high. The four criteria learned from the physical study of the biological material and reported in Fig. $2 \mathrm{a}$ as essential to claim the biomimicry status of the sample include the monolayer status, 3D structural continuity, 2D color discontinuity, and transverse pitch gradient. The biomimetic sample is chemically a monocomponent, while the cuticle is a chitin protein composite. The compaction can be taken as high in the cuticle and as medium in the biomimetic sample because the combination of common physical properties occurs within thicknesses equal to ca. 15 and $40 \mu \mathrm{m}$, respectively. Two characteristics are clearly different: reflection colors are vibrant in the cuticle and much less vibrant in the biomimetic sample, and polygonal cells are concave in the cuticle and convex in the biomimetic sample.

Biomimetic applications in optical communication. The twisted CLC patterns issued from the present design pave the way to many possibilities of practical uses.

They may inspire future applications in optics, in the field of non-specular properties such as deflection and lensing in geometric phase planar optics ${ }^{66-68}$. The phase of light that is Bragg-reflected off the helical structure can be controlled over $0-2 \pi$ depending on the spatial phase of the helical structure ${ }^{66}$. The manufacture of planar elements with arbitrary reflected wave fronts via orientation control is thus desired. This requires the helix phase to vary depending on position, which objective is reached with on-demand chiral patterns provided by the present design.
Insects and humans have common concerns in terms of energy regulation, optical communication and camouflage ${ }^{69}$. Concerning the last two functions, the fabrication of biomimetic samples inspires us to design tags in the field of cryptography and encryption ${ }^{70,71}$. Graphically and spectrally encoded tags have become appealing for carrying information about tagged products because of their high coding capacity and wide applicability ${ }^{71}$. Incorporating anti-counterfeiting tags with physical unclonable functions (PUFs) into objects is a promising solution for their authentication. Ongoing research hints at future LC-based technologies that may benefit from the peculiar behavior of CLCs subject to curvature, as reported for the case of cholesteric shells and droplets with the objective of generating unclonable patterns ${ }^{72-74}$.

We provide two physical methods in relation to the size of the pattern to code and the technical solution to be implemented: the objective consists of encoding and hiding a signature (taggant) with different colors at different scales, from the millimetre to micrometre scale (Fig. 4), with an optical readout visible to the naked eye (Fig. 4a) or by optical microscopy (Fig. 4b).

For millimetre-size taggants (Fig. 4a), the free surface of the green film is signed by the user with a pencil dipped into a polymer (PVA) solution. After water evaporation, the signature is invisible to the naked eye (Supplementary Fig. 8) and difficult to discern with the help of a microscope since the polymer film is thin, isotropic and transparent. It is revealed after thermal treatment $\left(120^{\circ} \mathrm{C}\right.$ for $\left.1 \mathrm{~h}\right)$ as the consequence of the nucleation and growth of the polygonal texture in the polymer-free parts of the film. The difference between the planar ("Joe" signature) and polygonal textures leads to color differences. Micrometre-size taggants (Fig. 4b) can be formed by fixing a copper grid to the film and depositing a polymer solution on the grid-film system. The grid is then removed, and the film is annealed. Grid patterns of taggants with geometrical features at different scales, from several tens of micrometres to a few micrometres, are revealed after thermal treatment and can be achieved by using different spin-coating conditions to deposit the polymer solution (see Methods), or grids with different geometrical features. Glassforming CLCs are easily malleable, and their structure can be locked in solid paint after quenching. Solidified hybrid textures can be formed on a support that can be safely applied on an object by printing it on a plastic film or embedding it in a resin. Under illumination, the tags reveal patterns that, according to the taggant size, are visible to the naked eye, without or with the use of polarizers, or detectable by optical microscopy. The hybrid planar-polygonal texture provides the possibility of forming an image (the PUF tag) when it is embedded in a polygon-textured matrix, producing a specific optical response (the PUF pattern), 
which has to be recorded in a list of PUF keys (a database). An assembly of cells carries an additional level of PUF since the relative positions, sizes and optical patterns of cells are unique, not clonable and unpredictable. C. gloriosa may display information and hide in the background. Therefore, the comparable role of artificial tags and biological patterns brings an additional criterion to the biomimetic nature of the present biomimetic samples in terms of physical functions.

By using CLC oligomers we have engineered a single-layer material mimicking the striped cuticle of the scarab beetle $C$. gloriosa. Textural, structural and color properties were reproduced at several length scales. The functionalities of both materials were relevant to the topics of optical communication and camouflage. A multicriterion comparison revealed a very high level of biomimicry. The double versatility of colors and patterns was achieved during a simple and single stage based on a thermally driven self-organization process. The fabrication of biomimetic samples has inspired us to design proof-of-concept prototypes of optical tags in the field of cryptography and encryption. The design strategy involves an optimal use of resources in the spirit of eco-design, and a high versatility of chiral patterns unreached by the current manufacturing techniques.

\section{Methods}

Biological samples. A male specimen was collected at Madera Canyon (Santa Cruz County, Arizona, USA) in July 2014. The cuticle samples including green and silver bands correspond to the elytra and were removed with a razor blade.

Synthetic materials. LC vitrifiable side-chain oligomers with a siloxane backbone were obtained from Wacker-Chemie GmbH, Munich, Germany (Supplementary Fig. 3).

\begin{abstract}
Alignment and sacrificial layers used in the manufacture of cholesteric films. A PVA layer formed on the glass substrates supporting the cholesteric films was used as a promoter of the Grandjean planar texture or as a sacrificial layer to obtain free-standing films after the sample was placed in water. PVA as a powder (P8136, Sigma-Aldrich) was dissolved at $4 \mathrm{wt} . \%$ in distilled water. The aqueous solution was deposited on the substrate using a spin coater (Ossila Ltd.). The thickness of the dried film was $\sim 2 \mu \mathrm{m}$. A single sequence of spin-coating consisted of the deposition of $1.30 \mathrm{ml}$ of solution during a program consisting of three sequences with the following speed rates and durations: 5000 rpm@20s-4000 rpm@40s3000 rpm@60 s. This operation was repeated three times.
\end{abstract}

Preparation of individual films for the biomimetic samples. Two distinct greenreflecting (SG) and infrared-reflecting (IR) samples were prepared on a heating stage at $120^{\circ} \mathrm{C}$. SG was used as provided by the supplier. IR was a blend made with $40 \mathrm{wt} . \%$ of a red-reflecting compound (SR) and $60 \mathrm{wt} . \%$ of a nematic compound (SN). A 50- $\mu \mathrm{m}$-thick film of SG was coated with a razor blade on a $150-\mu \mathrm{m}$-thick cover plate. The sample was then quenched at room temperature (RT). The mean reflection wavelength of the film was $520 \mathrm{~nm}$ (Supplementary Fig. 7). A 20- $\mu \mathrm{m}-$ thick IR film was sandwiched between a 1-mm-thick glass plate and a $150-\mu \mathrm{m}$ thick cover plate separated by $20-\mu \mathrm{m}$-thick spacers. The sample was then quenched at RT. The mean reflection wavelength of the film was $1600 \mathrm{~nm}$ (Supplementary Fig. 7). The cover plate was sharply removed from the film with the aid of pressurized air to avoid the formation of scratches at the surface of the film. That the free surface of the film remained smooth after the operation was checked by optical microscopy. When the realization of free-standing samples was the goal, the glass plate that supported the IR film was previously covered with an $80-\mu \mathrm{m}$-thick PVA film made from a $30 \mathrm{wt} . \%$ solution. The sample was then placed in a vial of distilled water. It detached from the plate in $<1 \mathrm{~h}$ at RT. The PVA bands on the top surface also dissolved in water. The sample thus consisted of a monolithic piece of glassy polymer that did not include any foreign material.

Formation of bands in the biomimetic samples. To draw bands, an alternate solution to the use of a pencil dipped in PVA solution was to place $75-\mu \mathrm{m}$-thick dicing tape (6033, Loadpoint), cut with dimensions close to those of the green bands of C. gloriosa, on the free surface of the bilayer film (Supplementary Fig. 9), at the end of stage $n^{\circ} 3$ in Fig. 2b. The surface of the sample with tape (typical size: $1.5 \mathrm{~cm}^{2}$ ) was covered with $1.30 \mathrm{ml}$ of PVA solution and spin-coated according to a program consisting of three sequences with the following speed rates and durations: 5000 rpm@20 s-4000 rpm@40 s-3000 rpm@60 s. This operation was repeated three times. The tape was gently removed from the film with the help of tweezers before the PVA film was completely dry.

Preparation of functional films for optical cryptography at the micrometre scale. A copper grid (1GC 200 Pelco, Ted Pella) with square patterns (hole width $=90 \mu \mathrm{m}$, bar width $=37 \mu \mathrm{m}$ ) was fixed with adhesive tape on a $20-\mu \mathrm{m}$-thick SG open film. A quantity of $1.30 \mathrm{ml}$ of PVA solution was spin-coated on the free surface of the film at $900 \mathrm{rpm}$ for $6 \mathrm{~s}$ for a variable number of sequences, therefore obtaining a variable quantity of solution. The promotion of one type of pattern size at the detriment of other size depended on this process. The thicknesses of the thread patterns (related to the grid bars-the solution was trapped by capillarity below them) with mean sizes of 35,6 and $2 \mu \mathrm{m}$ (Fig. $4 \mathrm{~b}$ ) corresponded to 6,4 and 3 sequences. The grid was then removed, and the sample was annealed at $120^{\circ} \mathrm{C}$ for $1 \mathrm{~h}$.

Inclusions of samples for ultramicrotomy. A small piece of material was embedded in EMBed-812 resin (Electron Microscopy Sciences), which was then cured at $62{ }^{\circ} \mathrm{C}$ for 2 days (biological sample) or at $37^{\circ} \mathrm{C}$ for 11 days (biomimetic sample).

Preparation of TEM samples by ultramicrotomy. A diamond knife at ambient temperature was used to cut 80 -nm-thick ultrathin slices with a Leica UCT ultramicrotome. The material was cut perpendicular to the film surface (cross sections). Slices were placed on single-slot Formvar-coated copper grids (GS2x1C3, Gilder Grids Ltd.)

TEM conditions. A Jeol JEM-1400 microscope operating at $80 \mathrm{kV}$ equipped with a Gatan Orius SC1000B CCD camera was used.

Periodicity profiles from TEM images. Quantitative image analysis was performed with DigitalMicrograph software from Gatan. The intensities of the gray levels on a given surface of the images were analysed using the Profile option.

Optical micrographs. Textures were photographed with an Olympus BX51 stereomicroscope equipped with an Olympus DP73 digital camera.

Optical measurements. The reflectance and transmittance spectra of materials were collected at RT and normal incidence with unpolarized incident light. An HR2000CG-UV-NIR spectrophotometer from Ocean Optics (reflectance spectra) or a Cary 5000 UV-Vis-NIR spectrometer from Varian (transmittance spectra) was used. Baselines were recorded with air (resp. a mirror) in the beam path for transmittance (resp. reflectance) measurements.

Reporting summary. Further information on research design is available in the Nature Research Reporting Summary linked to this article.

\section{Data availability}

The data supporting the findings of this study are available within the paper and its Supplementary Information file and from the corresponding author upon reasonable request.

Received: 7 February 2020; Accepted: 24 July 2020;

Published online: 14 August 2020

\section{References}

1. Ball, P. The Self-Made Tapestry-Pattern Formation in Nature (Oxford University Press, Oxford, 1999).

2. Lee, D. Nature's Palette-The Science of Plant Colour (The University of Chicago Press, Chicago, 2007).

3. Kolle, M. \& Lee, S. Progress and opportunities in soft photonics and biologically inspired optics. Adv. Mater. 30, 1702669 (2018).

4. Yang, Y. et al. Recent progress in biomimetic additive manufacturing technology: from materials to functional structures. Adv. Mater. 30, 1706539 (2018).

5. Wegst, U. G. K., Bai, H., Saiz, E., Tomsia, A. P. \& Ritchie, R. O. Bioinspired structural materials. Nat. Mater. 14, 23-36 (2015).

6. Filippov, A. E., Kovalev, A. \& Gorb, S. N. Numerical simulation of the pattern formation of the springtail cuticle nanostructures. J. R. Soc. Interface 15, 20180217 (2018).

7. Lenau, T. \& Barfoed. M. Colours and metallic sheen in beetle shells-a biomimetic search for material structuring principles causing light interference. Adv. Eng. Mat. 10, 299-314 (2008). 
8. de Gennes, P. G. \& Prost, J. (eds) in The Physics of Liquid Crystals 263-329 (Oxford University Press, Oxford, 1993).

9. Snell-Rood, E. Bring biologists into biomimetics. Nature 529, 277-278 (2016).

10. Kaiser, N. \& Pulker, H. K. Optical Interference Coatings (Springer, New York, 2003).

11. Zhu, Y., Zhang, W. \& Zhang, D. Fabrication of sensor materials inspired by butterfly wings. Adv. Mater. Technol. 2, 160020 (2017).

12. Matranga, A. et al. Biomimetic reflectors fabricated using self-organising, selfaligning liquid crystal polymers. Adv. Mater. 25, 520-523 (2013).

13. Fernandes, S. N. et al. Mind the microgap in iridescent cellulose nanocrystal films. Adv. Mater. 29, 1603560 (2017).

14. Caveney, S. Cuticle reflectivity and optical activity in scarab beetles: the role of uric acid. Proc. R. Soc. Lond. B 178, 205-225 (1971).

15. Seago, A. E., Brady, P., Vigneron, J.-P. \& Schultz, T. D. Gold bugs and beyond: a review of iridescence and structural color mechanisms in beetles (Coleoptera). J. R. Soc. Interface 6, S165-S184 (2009).

16. Stavenga, D. K., Wilts, B. D., Leertouwer, H. L. \& Hariyama, T. Polarized iridescence of the multilayered elytra of the Japanese jewel beetle, Chrysochroa fulgidissima. Philos. Trans. B 366, 709-723 (2011).

17. Yu, K., Fan, T., Lou, S. \& Zhang, D. Biomimetic optical materials: integration of nature's design for manipulation of light. Prog. Mater. Sci. 58, 825-873 (2013).

18. Vignolini, S. et al. Pointillist structural color in Pollia fruit. Proc. Natl Acad. Sci. USA 109, 15712-15715 (2012).

19. Vignolini, S. et al. Structural color from helicoidal cell-wall architecture in fruits of Margarita nobilis. J. R. Soc. Interface 13, 20160645 (2016)

20. Pace, A. Cholesteric liquid crystal-like structure of the cuticle of Plusiotis gloriosa. Science 176, 678-680 (1972).

21. Sharma, V., Crne, M., Park, J. O. \& Srinivasarao, M. Structural origin of circularly polarized iridescence in jeweled beetles. Science 325, 449-451 (2009).

22. Fernandez del Rio, L., Arwin, H. \& Järrendahl, K. Polarizing properties and structural characteristics of the cuticle of the scarab beetle Chrysina gloriosa. Thin Solid Films 571, 410-415 (2014).

23. Fernandez del Rio, L., Arwin, H. \& Järrendahl, K. K. Polarization of light reflected from Chrysina gloriosa under various illuminations. Mat. Today.: Proc. 1S, 172-176 (2014).

24. Agez, G., Bayon, C. \& Mitov, M. Multiwavelength micromirrors in the cuticle of scarab beetle Chrysina gloriosa. Acta Biomater. 48, 357-367 (2017).

25. Bouchal, P. et al. Non-diffracting light in nature: anomalously reflected selfhealing Bessel beams from jewel scarabs. APL Photon 4, 126102 (2019).

26. Kelker, H. \& Hatz, R. (eds) in Handbook of Liquid Crystals 293-337 (Verlag Chemie, Weinheim, 1980)

27. Hernández-Jiménez, M. et al. Qualitative correlation between structural chirality through the cuticle of Chrysina aurigans scarabs and left-handed circular polarization of the reflected light. Opt. Mater. Express 4, 2632-2645 (2014).

28. Libby, E. et al. Light reflection by the cuticle of $C$. aurigans scarabs: a biological broadband reflector of left handed circularly polarized light. J. Opt. 16, 082001 (2014).

29. Thomas, B., Seago, A. \& Robacker, D. C. Reflections on golden scarabs. Am. Entomol. 53, 224-230 (2007)

30. Azofeifa, D. E. et al. A quantitative assessment approach of feasible optical mechanisms contributing to structural color of golden-like Chrysina aurigans scarab beetles. J. Quant. Spectrosc. Rad. Transf. 160, 63-74 (2015).

31. Kolle, M. et al. Mimicking the colorful wing scale structure of the Papilio blumei butterfly. Nat. Nanotech. 5, 511-515 (2010).

32. Mark, E. \& Lin, G. The Polysiloxanes (Oxford University Press, Oxford, 2015).

33. Kreuzer F.-H. et al. in Organosilicon Chemistry III (eds Auner, N. \& Weiss, J.) 566-586 (Wiley-VCH, Weinheim, 1997).

34. Bunning, T. J. \& Kreuzer, F.-H. Cyclosiloxane-based liquid crystalline materials. Trends Polym. Sci. 3, 318-323 (1995).

35. Eberle, H.-J., Miller, A. \& Kreuzer, F.-H. Inverse angle dependence of the reflection colors of cholesteric polymeric liquid crystals mixed with pigments. Liq. Cryst. 5, 907-916 (1989)

36. Tsai, M. L., Chen, S. H. \& Jacobs, S. D. Optical notch filter using thermotropic liquid crystalline polymers. Appl. Phys. Lett. 54, 2395 (1989).

37. Mitov, M., Binet, C., Boudet, A. \& Bourgerette, C. Glassy cholesteric broadband reflectors with a pitch gradient: material design, optical properties and microstructure. Mol. Cryst. Liq. Cryst. 358, 209-223 (2001).

38. Etienne, S., David, L., Mitov, M., Sixou, P. \& Ngai, K. L. Mechanical spectroscopy of side-chain liquid crystalline polymers in the glass transition range. Macromolecules 28, 5758-5764 (1995).

39. Korenic, E. M., Jacobs, S. D., Faris, S. M. \& Li, L. Color gamut of cholesteric liquid-crystal films and flakes by standard colorimetry. Colour. Res. Appl. 23, 210-220 (1998).

40. Trajkovska, A., Varshneya, R., Kosc, T. Z., Marshall, K. L. \& Jacobs, S. D. Enhanced electro-optic behavior for shaped polymer cholesteric liquid-crystal flakes made using soft lithography. Adv. Funct. Mater. 15, 217-222 (2005).
41. Shibaev, P. V. et al. Color changing and lasing stretchable cholesteric films. Opt. Express 16, 2965-2970 (2008).

42. Shibaev, P. V. in Liquid Crystal Microlasers (eds Blinov, L. M. \& Bartolino, R.) 127-140 (Transworld Research Network, Kerala, 2010).

43. Mitov, M., Portet, C., Bourgerette, C., Snoeck, E. \& Verelst, M. Long range structuring of nanoparticles by mimetism with a cholesteric liquid crystal. Nat. Mater. 1, 229-231 (2002).

44. Bitar, R., Agez, G. \& Mitov, M. Cholesteric liquid crystal self-organization of gold nanoparticles. Soft Matter 7, 8198-8206 (2011).

45. Neradovskiy, M., Scarangella, A., Jullien, A. \& Mitov, M. Dispersion of $20 \mathrm{fs}$ pulses through band edges of cholesteric liquid crystals. Opt. Express 27, 021794 (2019)

46. Bouligand, Y. Recherches sur les textures des états mésomorphes-Les champ polygonaux dans les cholestériques. J. Phys. 33, 715-736 (1972).

47. Saupe, A. Disclinations and properties of the directorfield in nematic and cholesteric liquid crystals. Mol. Cryst. Liq. Cryst. 21, 211-238 (1973).

48. Bunning, T. J. et al. Cholesteric liquid crystals: image contrast in the TEM. Liq Cryst. 16, 769-781 (1994).

49. Meister, R., Dumoulin, H., Hallé, M.-A. \& Pieranski, P. The anchoring of a cholesteric liquid crystal at the free surface. J. Phys. II 6, 827-844 (1996).

50. Meister, R., Hallé, M.-A., Dumoulin, H. \& Pieranski, P. Structure of the cholesteric focal conic domains at the free surface. Phys. Rev. E 54, 3771-3782 (1996).

51. Bobrovsky, A., Mochalov, K., Chistyakov, A., Oleinikov, V. \& Shibaev, V. Features of double-spiral "valley-hills" surface topography formation in photochromic cholesteric oligomer-based films and their changes under polarized light action. Macromol. Chem. Phys. 213, 2639-2646 (2012).

52. Bobrovsky, A., Sinitsyna, O. V., Abramchuk, A., Yaminsky, I. \& Shibaev, V. Atomic force microscopy study of surface topography of films of cholesteric oligomer- and polymer-based mixtures with photovariable helix pitch. Phys. Rev. E 87, 012503 (2013).

53. Bayon, C., Agez, G. \& Mitov., M. Wavelength-tunable light shaping with cholesteric liquid crystal microlenses. Lab Chip 14, 2063-2071 (2014).

54. Sinitsyna, O. V., Bobrovsky, A. Y., Meshkov, G. B., Yaminsky, I. V. \& Shibaev V. P. Surface relief changes in cholesteric cyclosiloxane oligomer films at different temperatures. J. Phys. Chem. B 119, 12708-12713 (2015).

55. Bayon, C., Agez, G. \& Mitov Size-effect of oligomeric cholesteric liquid-crystal microlenses on the optical specifications. Opt. Lett. 40, 4763-4766 (2015).

56. Sinitsyna, O. V., Bobrovsky, A. Y., Meshkov, G. B., Yaminsky, I. V. \& Shibaev V. P. Direct observation of changes in focal conic domains of cholesteric films induced by ultraviolet irradiation. J. Phys. Chem. B 121, 5407-5412 (2017).

57. Jullien, A., Scarangella, A., Bortolozzo, U., Residori, S. \& Mitov, M. Nanoscale hyperspectral imaging of of cholesteric liquid crystals with oblique helicity. Soft Matter 15, 3256-3263 (2019).

58. Cui, Y., Zola, R. S., Yang, Y.-C. \& Yang, D.-K. Alignment layers with variable anchoring strengths from polyvinyl alcohol. J. Appl. Phys. 111, 063520 (2012).

59. Kumar, C. et al. Replicating the complexity of natural surfaces: technique validation and applications for biomimetics, ecology and evolution. Philos. Trans. Roy. Soc. A-Math. Phys. Eng. Sci. 377, 20180265 (2019).

60. Chigrinov, V. G., Kozenkov, V. M. \& Kwok, H.-S. Photoalignment of Liquid Crystalline Materials: Physics and Applications (John Wiley \& Sons, Ltd., Chichester, 2008).

61. Nie, Z. \& Kumacheva, E. Patterning surfaces with functional polymers. Nat. Mater. 7, 277-290 (2008).

62. van Oosten, C. L., Bastiaansen, C. W. M. \& Broer, D. J. Printed artificial cilia from liquid-crystal network actuators modularly driven by light. Nat. Mater. 8, 677-682 (2009).

63. Andersen, S. O. in Insect Molecular Biology and Biochemistry Ch. 6 (ed. Gilbert, L. I.) (Academic Press, London, 2012).

64. Zografopoulos, D. C., Kriezis, E. E., Mitov, M. \& Binet, C. Theoretical and experimental optical studies of cholesteric liquid crystal films with thermally induced pitch gradients. Phys. Rev. E 73, 061701 (2006).

65. St. John, W. D., Fritz, W. J., Lu, Z. J. \& Yang, D.-K. Bragg reflection from cholesteric liquid-crystals. Phys. Rev. E 51, 1191-1198 (1995).

66. Kobashi, J., Yoshida, H. \& Ozaki, M. Planar optics with patterned chiral liquid crystals. Nat. Photon 10, 389-392 (2016).

67. Chen, P. et al. Chirality invertible superstructure mediated active planar optics. Nat. Commun. 10, 2518 (2019).

68. Ma, L.-L. et al. Light-activated liquid crystalline hierarchical architecture toward photonics. Adv. Optical Mater. 7, 1900393 (2019).

69. Mitov, M. Cholesteric liquid crystals in living matter. Soft Matter 13, 4176-4209 (2017).

70. Pappu, R., Recht, B., Taylor, J. \& Gershenfeld, N. Physical one-way functions Science 297, 2026-2030 (2002).

71. Arppe, R. \& Sørensen, T. J. Physical unclonable functions generated through chemical methods for anti-counterfeiting. Nat. Rev. Chem. 1, 0031 (2017). 
72. Geng, Y. et al. High-fidelity spherical cholesteric liquid crystal Bragg reflectors generating unclonable patterns for secure authentication. Sci. Rep. 6, 26840 (2016).

73. Lenzini, G. et al. Security in the shell: an optical physical unclonable function made of shells of cholesteric liquid crystals. In Proc. 2017 IEEE Workshop on Information Forensics and Security (WIFS) 1-6 (eds Furon, T. \& Troncoso, C.) (IEEE, 2017)

74. Schwartz, M. et al. Cholesteric liquid crystal shells as enabling material for information-rich design and architecture. Adv. Mater. 30, 1707382 (2018).

75. Zhang, Y., Paris, O., Terrill, N. J. \& Gupta, H. S. Uncovering threedimensional gradients in fibrillar orientation in an impact-resistant biological armour. Sci. Rep. 6, 26249 (2016).

76. Mitov, M., Soldan, V. \& Balor, S. Observation of an anisotropic texture inside the wax layer of insect. Arthropod Struct. Dev. 47, 622-626 (2018).

77. Blomquist, G. J. \& Bagnères, A.-G. Insect Hydrocarbons-Biology, Biochemistry and Chemical Ecology (Cambridge University Press, Cambridge, 2010).

\section{Acknowledgements}

M.M. thanks the Agence Nationale de la Recherche (France) for financial support (Grant: ANR-17-CE30-0025) and Dr. E. Hanelt from Wacker-Chemie GmbH (Munich, Germany) for providing him with oligomers.

\section{Author contributions}

M.M. led the conceptual development of the research, oversaw data collection, performed the analysis of results, organized the contents of the manuscript, prepared the figures and wrote the manuscript. A.S. contributed to the conceptual development of samples, carried out the manufacture of biomimetic samples along with their optical characterization, and participated in the analysis of results. V.S. carried out the conceptual development of operations related to the TEM investigations, cut the inclusions by ultramicrotomy and carried out TEM observations. M.M. prepared the inclusions for TEM observation and carried out the conception and manufacture of samples for cryptography. All authors revised the manuscript.

\section{Competing interests}

The authors declare no competing interests.

\section{Additional information}

Supplementary information is available for this paper at https://doi.org/10.1038/s41467020-17884-0.

Correspondence and requests for materials should be addressed to M.M.

Peer review information Nature Communications thanks Huai Yang, Jan Lagerwall and Seungwoo Lee for their contribution to the peer review of this work.

Reprints and permission information is available at http://www.nature.com/reprints

Publisher's note Springer Nature remains neutral with regard to jurisdictional claims in published maps and institutional affiliations.

(c) Open Access This article is licensed under a Creative Commons Attribution 4.0 International License, which permits use, sharing, adaptation, distribution and reproduction in any medium or format, as long as you give appropriate credit to the original author(s) and the source, provide a link to the Creative Commons license, and indicate if changes were made. The images or other third party material in this article are included in the article's Creative Commons license, unless indicated otherwise in a credit line to the material. If material is not included in the article's Creative Commons license and your intended use is not permitted by statutory regulation or exceeds the permitted use, you will need to obtain permission directly from the copyright holder. To view a copy of this license, visit http://creativecommons.org/ licenses/by/4.0/.

(C) The Author(s) 2020 